\title{
Potential energy landscape and long time dynamics in a simple model glass
}

\author{
L. Angelani ${ }^{1}$, G. Parisi $^{2}$, G. Ruocco ${ }^{1}$ and G. Viliani ${ }^{3}$, \\ 1 Universitá di L'Aquila and Istituto Nazionale di Fisica della Materia, I-67100, L'Aquila, Italy. \\ 2 Universitá di Roma La Sapienza and Istituto Nazionale di Fisica Nucleare, I-00185, Roma, Italy. \\ ${ }^{3}$ Universitá di Trento and Istituto Nazionale di Fisica della Materia, I-38050, Povo, Trento, Italy.
}

(April 4, 2017)

\begin{abstract}
We analyze the properties of a Lennard-Jones system at the level of the potential energy landscape. After an exhaustive investigation of the topological features of the landscape of the systems, obtained studying small size sample, we describe the dynamics of the systems in the multi-dimensional configurational space by a simple model. This consider the configurational space as a connected network of minima where the dynamics proceeds by jumps described by an appropriate master equation. Using this model we are able to reproduce the long time dynamics and the low temperature regime. We investigate both the equilibrium regime and the off-equilibrium one, finding those typical glassy behavior usually observed in the experiments such as: i) stretched exponential relaxation, ii) temperature-dependent stretching parameter, iii) breakdown of the Stokes-Einstein relation, and $i v$ ) appearance of a critical temperature below which one observes deviation from the fluctuation-dissipation relation as consequence of the lack of equilibrium in the system.
\end{abstract}

PACS Numbers : 61.20.Lc, 64.70.Pf, 82.20.Wt

\section{INTRODUCTION}

The landscape paradigm [1] is a very useful point of view for the study of glassy systems. The detailed analysis of the free energy or potential energy surfaces allows us to get insight into the rich phenomenology exhibited by glass-forming liquids in the supercooled phase, around the glass transition region and in the low temperature glassy regime. While the investigation of the free energy surface is a very hard task starting from the microscopic description of the system, since the landscape details are very strongly temperature dependent, the description of the potential energy landscape is a much more tractable problem, and is a good starting point to investigate properties at not-too-high temperatures. The trajectory of the representative point of the system in the configurational phase space can be viewed as a path in the multidimensional potential energy surface. The dynamics is strongly influenced by the topography of the landscape: local minima, barriers height, attraction basins and further topological features. In recent years it has been show that the details of the potential energy surface are of great importance in determining the properties of many systems exhibiting glassy behavior, like glass-forming liquids, protein folding, atomic cluster or evolutionary biological models.

In this paper we numerically investigate the low temperature dynamical properties of a simple system, i.e. a monoatomic Lennard-Jones system, through an analysis of its multidimensional potential energy surface and a simple model for the loe temperature dynamics. In the first part (Section II), by studying small sample size, we give a comprehensive description of the potential energy landscape of the systems: minima, barriers, reaction paths, saddle points, and determine statistical distributions and cross-correlations among the analyzed quanti- ties. In the second part (Section III) we use this information to set up a simple model for the study of the long-time relaxation dynamics of the system; the model consists of a connected network of potential energy minima with a jump dynamics described by an appropriate master equation. This allows us to get information on the behavior of the system for times long enough that a direct Molecular Dynamics (MD) simulation is not feasible [2]. After a static (thermodynamical) test of the model, we determine the dynamical equilibrium properties and also the off-equilibrium ones, and discuss the results. In the last Section we report the conclusions.

\section{POTENTIAL ENERGY LANDSCAPE}

We numerically investigate the topology of the potential energy hypersurface of a Lennard-Jones $6-12$ system of $N$ interacting particles in a cubic box with periodic boundary conditions. The pair potential is:

$$
V_{L J}(r)=4 \epsilon\left[\left(\frac{\sigma}{r}\right)^{12}-\left(\frac{\sigma}{r}\right)^{6}\right],
$$

with $r$ the Euclidean distance between two particles. The physical parameters $\sigma$ and $\epsilon$ are choose to describe an Argon system: $\sigma=0.3405 \mathrm{~nm}$ and $\epsilon / K_{B}=125.2 \mathrm{~K}\left(K_{B}\right.$ is the Boltzmann constant). In order to have an exhaustive description of the energy landscape, we investigate small size systems, with a number of particles $N<30$. As we shall see later, such small systems can nevertheless exhibit complex enough behavior.

Due to the small size of the system, the range of interactions among the particles is of the same order as the box length. It is then much more appropriate to use a multi-image method instead of the usual minimum image method [3], in which each particle interacts only with 
the nearest image (generated by the periodic boundary conditions) of all other particles. In our case many images contribute, and it is necessary to consider them in the calculation of the interactions. The choice we made introduces an angular dependence in the pair potential which, however, is negligible with respect to the radial one. The simulated density is $\rho=4.2 \cdot 10^{-2} \mathrm{~mol} / \mathrm{cm}^{3}$, which was obtained by Demichelis et al. [5] as the smallest value at which a Lennard-Jones system of $N=864$ Argon particles is stable in the glassy state after a rapid quench from high temperature.

\section{A. Minima}

As a first step we search for the potential energy minima. We used a modified conjugate gradient method starting from high temperature configurations obtained during a MD simulation. In this way we find the so called "inherent configurations", corresponding to local minima. New minima are identified by their potential energy values. We analyzed systems with $N=11 \div 29$ and, for each $N$, we stopped the search when the rate at which new low energy minima are found (for example in lowest first third of the full energy range) is smaller than a given number (about $10^{-4}$ ). In this way we were able to obtain a good classification of the low energy minima. The number of found minima, $\mathcal{N}$, does not show a clear and well defined dependence on $N$, contrary to the case of small clusters [6], so that it is not possible to give an estimate of the coefficient $\alpha$ in the expected exponential growth: $\mathcal{N} \propto \exp (\alpha N)$ (for clusters $\alpha \sim 1$ ). We observe indeed for some values of $N$ a strong tendency of the system to fall always in the same minima. In this case an exhaustive research of the inherent structures is a very hard numerical task. this explains the not clear dependence found.

Once most minima are classified, we analyze their features: energy, static structure factor, curvature and distances.

\section{Energy}

The first important information about an inherent configuration is its potential energy value. From the energy distribution it is sometimes possible to recognize the crystalline-like configurations. Usually one finds an evident gap between the lowest energy minima, the crystalline-like ones, and the other ones with higher energies, as can be seen in Fig. 1 where we report the distribution of minima energies per particle for $N=29$. In other cases the situation is not so clear and it is useful to use a different method to characterize the nature of an inherent configuration; the most useful quantity in determining the spatial order structure of a minimum is its static structure factor, which allows (imperfect) crystallike configuration also of high energy to be identified.

\section{Static structure factor}

In order to classify the spatial distribution of particle in a given minimum of the potential energy we use the static structure factor:

$$
\hat{S}(\vec{q})=\frac{1}{N}\left|\sum_{j} \exp \left(i \vec{q} \cdot \vec{r}_{j}\right)\right|^{2} .
$$

Due to the finite size of the system the allowed $\vec{q}$ vectors are of the form $\vec{q}=(2 \pi / L) \vec{n}$, with $\vec{n}=\left(n_{x}, n_{y}, n_{z}\right)$ an integer vector. We define the quantity

$$
S(q)=\frac{1}{n_{q}} \sum_{\vec{q} \in\{q, \Delta q\}} \hat{S}(\vec{q}),
$$

where $\sum_{\vec{q} \in\{q, \Delta q\}}$ is a sum over the $n_{q}$ vectors with modulus within $q \pm \Delta q$. For a pure crystalline configuration of $N$ particles $S(q)$ consists of Bragg peaks, and its value at the peaks is $S_{\max }=N$. For amorphous configurations $S(q)$ does not present a well defined peak structure and the highest value is $S_{\max } \sim 2 \div 3$, obtained for a $q$ value of the order of the inverse mean distance of two near particles. For small sized systems, like those analyzed here, there are intermediate situations and we use the criterion $S_{\max }<N / 2$ to determine the amorphous nature of an inherent structure.

\section{Curvature}

Another important property of a minimum is its overall curvature $c$, defined as the determinant of the Hessian $\Phi^{\prime \prime}$ of the potential energy function $\Phi$ :

$$
c=\operatorname{det}\left(\Phi^{\prime \prime}\right)
$$

The eigenvalues of the Hessian matrix are proportional to the squared vibrational eigenfrequencies. In the inset of Fig. 1 we show the distribution of

$$
\gamma=\frac{1}{3 N-3} \log _{10}(c / m)^{1 / 2},
$$

where $m$ is the mass of the particles (we use the Argon's value $m=40 \mathrm{amu}$ ). The quantity $\gamma$ is thus proportional to the sum of the logarithms of the frequencies of normal vibrational modes

$$
\gamma=\frac{1}{3 N-3} \sum_{\alpha} \ln \omega_{\alpha},
$$

with $\alpha=1, \ldots, N-3$ (the three zero frequencies corresponding to rigid translations have been eliminated from the sum). As can be seen in Fig. 1, the highest $\gamma$ values correspond to the minima with lowest energy, i.e. the crystalline-like minima which are narrower and deeper than the other packing structures (see also Sec. II-C). 


\section{Stress tensor}

For each minimum we determined the off-diagonal part of the microscopic stress tensor:

$$
\left\{\begin{array}{l}
\sigma^{z x}=-\sum_{i<j} V_{L J}^{\prime}\left(r_{i j}\right) \frac{z_{i j} x_{i j}}{r_{i j}} \\
\sigma^{x y}=-\sum_{i<j} V_{L J}^{\prime}\left(r_{i j}\right) \frac{x_{i j} y_{i j}}{r_{i j}} \\
\sigma^{y z}=-\sum_{i<j} V_{L J}^{\prime}\left(r_{i j}\right) \frac{y_{i j} z_{i j}}{r_{i j}}
\end{array}\right.
$$

where $x_{i j}, y_{i j}, z_{i j}$ are the components of $\vec{r}_{i j}=\vec{r}_{i}-\vec{r}_{j}$; these quantities will be useful in determining the shear viscosity. The form of the stress tensor we use is the $q=0$ extrapolation of the $q$-dependent expression []]

$$
\begin{aligned}
\sigma_{\alpha, \beta}(\vec{q})= & \sum_{i}\left[m v_{i, \alpha} v_{i, \beta}-\sum_{i<j} \frac{r_{i j, \alpha} r_{i j, \beta}}{r_{i j}^{2}} P_{q}\left(r_{i j}\right)\right] . \\
& \cdot \exp \left(i \vec{q} \cdot \vec{r}_{i}\right)
\end{aligned}
$$

with

$$
P_{q}(r)=r V_{L J}^{\prime}(r) \frac{1-\exp (i \vec{q} \cdot \vec{r})}{i \vec{q} \cdot \vec{r}} .
$$

The index $i$ and $j$ refer to particles, while $\alpha$ and $\beta$ label the spatial axes $x, y, z$. In (7) we have omitted the kinetic term, not well defined in an inherent configuration; our hypothesis is that also this truncated form is able to well describe the relaxation processes.

\section{Distances}

We now turn to the relationships among the minima; in particular we determined the mutual distances in the $3 \mathrm{~N}$-dimensional configuration space; in this regard it is important to take into account all the symmetry operations of the problem. Indicating with $\underline{r}_{a}=\left(\vec{r}_{1}^{a}, \ldots, \vec{r}_{N}^{a}\right)$ the $3 N$ coordinates of the particles in the minimum $a$, we define the distances $d_{a b}$ between minima $a$ e $b$ :

$$
d_{a b}=\min _{T, R, \pi}\left(\left|\underline{r}_{a}-\underline{r}_{b}\right|\right),
$$

where the minimization is made with respect to the continuous translations $(T)$, discrete rotations and reflections $(R)$, and permutations $(\pi)$ of the particles.

The minimization over the continuous translations $(T)$ is done by putting, sequentially, each particle of $a$ in the same place as each one of $b$. The minimization over the rotations and reflections $(R)$ is carried out by considering the 48 symmetry operations of the cubic group; the minimization over particle permutation $(\pi)$ is apparently an hard task to solve, as one should consider all the $N$ ! possible configurations in a direct calculation, but this is not the case actually. The problem is of polynomial type, i.e. the time needed to find the optimum solution grows as a polynomial function of the size, $N$, of the system (on the other hand non-polynomial problems require an exponential computational time, i.e. the travel salesman problem). The optimization problem we need to solve is a bipartite matching problem, which can be done in very short computational time by using an appropriate algorithm.

\section{B. Barriers}

A very important topological quantity in determining the dynamical behavior of the system is the energy barrier experienced by the system in traveling from one minimum to another. At first sight it might seem that it is accurate enough to evaluate the barrier along the straight path joining two minima in the $3 N$-dimensional space. However, as evidenced by Demichelis et al. [5], this produces in most cases much higher barriers than an evaluation along the least action path, indicating that the straight path approximation is often not good. We have then determined the least action path for each pair of minima $a$ and $b$, which is defined as the path that minimizes the action functional

$$
S[\ell]=\int_{\ell} d s \sqrt{2 m\left[\Phi(\underline{r}(s))-\Phi_{0}\right]},
$$

where $\ell$ is a generic path between the minima, $s$ is the curvilinear coordinate and $\Phi_{0}=\min \left\{\Phi_{a}, \Phi_{b}\right\}$. This functional problem is simplified by dividing the path into a finite number of intervals (typically we use $n=16$ intervals) and by minimizing the action function with respect to the extreme of the $n$ segments constrained to move in hyperplanes perpendicular to the straight path. The highest potential-energy value along the least action path determines the barrier height and identifies the saddle point.

We have only analyzed the system with $N \leq 17$, due to the very long computational times needed in the cases with $N>17$ where there are too many pairs of minima to take into account. We report the results for the largest system analyzed, $N=17$ with $\mathcal{N}=38$ minima. In Fig. 2 we show an example of the potential energy profile along the straight path (dashed line) and along the least action path (full line) between two minima. It is evident that the energy barrier is significantly lower in the latter case, although the two paths are not very distant in configuration space. Similar results are obtained for the other analyzed paths. Sometimes it happens that two minima are not directly connected, in the sense that the least action path joining them crosses a third minimum, and a non trivial connectivity among the minima emerges (in 
the analyzed system we find that each minimum on an average is directly connected to 20 other minima). For each saddle point along the least action path we determine the main properties: energy, curvature, down "frequency". In Fig. 3 is reported the energy distribution of the barriers $\Delta \Phi_{b a r .}$. The curvature is defined as the absolute value of the determinant of the Hessian of the potential energy evaluated at the saddle:

$$
c_{\text {sad. }}=\left|\operatorname{det} \Phi_{\text {sad. }}^{\prime \prime}\right|,
$$

In the inset of Fig. 3 we show the distribution of the quantity

$$
\gamma_{\text {sad. }}=\frac{1}{3 N-3} \log _{10}\left(c_{\text {sad. }} / m\right)^{1 / 2} .
$$

The down "frequency" $\tilde{\omega}_{\text {sad. }}$ is defined as the square root of the absolute value of the down curvature along the least action path:

$$
\tilde{\omega}_{\text {sad. }}^{2}=-\frac{\underline{v} \Phi_{\text {sad. }}^{\prime \prime} \underline{v}}{|\underline{v}|^{2}},
$$

where $\underline{v}$ is the tangent vector to the least action path at the saddle point.

\section{Correlations}

In order to have a full statistical description of the potential energy landscape, it is useful to investigate, besides the distributions of the different quantities, also their cross-correlations. We have then determined the linear correlation coefficient

$$
r(x, y)=\frac{\sum_{i}\left(x_{i}-\bar{x}\right)\left(y_{i}-\bar{y}\right)}{\left[\sum_{i}\left(x_{i}-\bar{x}\right)^{2} \sum_{j}\left(y_{j}-\bar{y}\right)^{2}\right]^{1 / 2}}
$$

for all the measured quantities $x$ and $y$. In Table I are reported the values obtained, together with the log $-\log$ correlations, in order to evidence possible power laws. In the first column we report the number of particles of the system analyzed (only for $N=17$ we have determined the least action paths and all the related quantities). It emerges that, substantially, energy difference and distance among the minima are not correlated, indicating that the topological structure of the inherent configurations is not energy-correlated. A weak correlation is observed between energy and curvature at stationary points (minima and saddle points). In Fig. 4 we show the cross-correlation between the energies of the minima and their curvatures. An interesting correlation is observed between barrier energies $\Delta \Phi_{b a r}$. and distances among minima $d_{a b}$ (Fig. 5), with a nearly linear correlation in double log scale (line in the figure).

We conclude the analysis of the energy landscape by determining the entropy ratio $R$, defined as the ratio between the curvature of saddle points and that of the related minima

$$
R=\frac{\left|\operatorname{det} \Phi_{\text {sad. }}^{\prime \prime}\right|}{\operatorname{det} \Phi_{\text {min. }}^{\prime \prime}}
$$

This quantity gives a quantitative measure of the ability of the system in finding the right path to reach another minimum. If $R \sim 1$ there are no entropic hindrances, while if $R \gg 1$ these effects become relevant, as the narrowness (higher value of the curvature) at the saddle makes the least action path toward that specific minimum unfavorable with respect to other escape route. For all the minimum-saddle-minimum triplets we have evaluated $R$; the majority of the values is in the range $10^{-2} \div 10$, in qualitative agreement with the results found in Lennard-Jones clusters [6].

\section{MODEL FOR THE DYNAMICS}

The investigation of the properties of glass-former liquids at the level of the energy landscape, allows us to introduce some approximations in the dynamics of the system. We define a simplified model which is able to capture the long time behavior of the system, and which consists of a connected network of potential energy minima with a jump dynamics among them described by an appropriate master equation [2].

The basic idea is quite simple. A glass is represented by a configurational point confined in a very small region of the accessible phase space and in the zero temperature limit (neglecting quantum effects) all the atoms are frozen in well defined positions, corresponding to some mechanically metastable state. When the temperature is raised jumps among different mechanically stable positions become possible. At finite and not-too-high temperature we assume the dynamically relevant processes are the following: a short time dynamics dominates by small vibrations around stable positions (this dynamics can be described within the harmonic approximation by diagonalizing the dynamical matrix), and a long time dynamics consisting of collective (involving many atoms) jumps among different stable positions. The main hypothesis we make is that there is a substantially clear separation of time scales between the two dynamical processes. This characterization of the dynamics is a good approximation at not-too-high temperature. By increasing the temperature, anharmonic effects become relevant to the vibration around the local minimum and, moreover, a clear time scale separation between fast vibrational and slow jumps dynamics is no longer possible. In a recent work [4] the validity of this hypothesis has been verified in a LennardJones binary mixture, giving a very good agreement with a direct MD investigation. To sum up, our model, which is expected to capture the physics of the system at low temperature, is based on two main hypotheses: 
1. clear cut difference between vibrational dynamics at short time and dynamics of collective jumps at long time;

2. description of the long time dynamics through a master equation, with the transition rates that depend on the topological properties of the potential surface.

The main advantages of the introduced model with respect to the usual MD computations are:

- we can avoid in a simple way the crystallization process, that always takes place in one component LJ systems, as we do not consider the crystalline minima in setting up the network.

- we can study in a direct way the low temperature properties, where usually the very long relaxation times require very long computational time. In MD the computational times are proportional to the physical times, while in the model introduced here the computational times are those needed to find the eigenvalues and eigenvectors of the transition matrix, independent of temperature;

- it is possible to evidence the relationships between the energy landscape and the behavior of the system.

To be more specific, the model is a connected network of potential energy minima and the master equation governing the jumps dynamics is:

$$
\frac{d p_{a}}{d t}\left(t ; b, t_{0}\right)=\sum_{c} W_{a c} p_{c}\left(t ; b, t_{0}\right),
$$

where $p_{a}\left(t ; b, t_{0}\right)$ is the probability that the system is at minimum $a$ at time $t$, if it was at minimum $b$ at time $t_{0}$. The off-diagonal elements of the matrix $W$ are the transition rates. The diagonal elements are fixed by the condition

$$
\sum_{a} W_{a c}=0
$$

In order to obtain an asymptotic behavior that reproduces the right Boltzmann weight the occupation probability must satisfy:

$$
\lim _{t \rightarrow \infty} p_{a}\left(t ; b, t_{0}\right)=p_{a}^{0} \equiv \frac{1}{\mathcal{Z}}\left(\operatorname{det} \Phi_{a}^{\prime \prime}\right)^{-1 / 2} \exp \left(-\beta \Phi_{a}\right),
$$

( $\mathcal{Z}$ is such that $\sum_{a} p_{a}^{0}=1$ and the pre-exponential factor follows from the harmonic vibration in each minimum), and the transition matrix $W$ must satisfy the detailed balance relation:

$$
W_{a b} p_{b}^{0}=W_{b a} p_{a}^{0} .
$$

The solution of the master equation is easily expressed in terms of eigenvalues $\lambda_{n}$ and eigenvectors $\alpha_{a}^{(n)}(n=$ $1, \ldots, M$, with $M$ matrix dimension) of $W$ :

$$
p_{a}\left(t ; b, t_{0}\right)=\left(p_{b}^{0}\right)^{-1} \sum_{n} \alpha_{a}^{(n)} \alpha_{b}^{(n)} \exp \left[\lambda_{n}\left(t-t_{0}\right)\right] .
$$

In the numerical calculus it is more convenient to express the solutions in terms of the eigenvectors of a new symmetric matrix $w_{a b}=W_{a b}\left(p_{b}^{0} / p_{a}^{0}\right)^{1 / 2}$ (whose eigenvalues coincide with those of $W$ ):

$$
p_{a}\left(t ; b, t_{0}\right)=\left(p_{a}^{0} / p_{b}^{0}\right)^{1 / 2} \sum_{n} e_{a}^{(n)} e_{b}^{(n)} \exp \left[\lambda_{n}\left(t-t_{0}\right)\right] .
$$

The model is well defined once we give an appropriate form to the transition matrix $W$. In order to determine the transition rates let us analyze the problem of escape from a metastable state; a useful point of view for systems with many degree of freedom is the description in term of a few relevant coordinates. This reduction is possible whenever there are few reaction coordinates with characteristic evolutionary times longer than those of the other degrees of freedom, which act as effective terms on the relevant coordinates, i.e. like noise and viscous terms. We suppose this is the case of our system whenever the temperature is not too high (the analysis of reaction paths made by Demichelis et al. [5] supports this hypothesis). Handling the problem as a MarkovianBrownian $d$-dimensional motion in the overdamped friction regime we obtain the form [8]

$$
W_{a b}=\frac{\tilde{\omega}_{\text {sad. }}^{2}}{\mu}\left[\frac{\operatorname{det} \Phi_{b}^{\prime \prime}}{\left|\operatorname{det} \Phi_{\text {sad. }}^{\prime \prime}\right|}\right]^{1 / 2} \exp \left[-\frac{\Phi_{\text {sad. }}-\Phi_{b}}{K_{B} T}\right],
$$

where $\tilde{\omega}_{\text {sad. }}$ is the down "frequency" at the saddle point and $\mu$ is a friction constant that determines the time scale (its value is fixed by a comparison with MD in the allowed temperature region).

All the characteristics of the model (properties of the connected network and parameters in the transition rates) are inferred from the computed properties of the potential energy landscape. We use the values of the $N=29$ system to determine local minima properties (energy, curvature, stress tensor) and those of $N=17$ system to determine connectivity properties (energy and curvature of saddle points, distances and connectivity among the minima). The values are extracted from the distribution found in the previous section in the following way: 
1. we extract $M$ energy values of the minima from the distribution of $N=29$ system (we exclude the crystalline-like configurations);

2. we assign to each minimum a value of curvature $c_{a}=\operatorname{det} \Phi_{a}^{\prime \prime}$ extracted from a bivariate distribution, thanks to the cross-correlation between energy and curvature; a stress tensor value is also extracted for each minimum;

3. for each minimum we randomly (in the analysis of the energy landscape we have found no correlation between energies and distances among minima) extract 20 minima connected to it, as obtained on an average for the system $N=17$;

4. we define a connection matrix $\kappa_{a b}$, containing the minimum steps, i.e. the number of minima crossed, necessary to go from $a$ to $b$; the distance matrix $d_{a b}$ is $\kappa_{a b}$ times the value extracted from the distribution of the distances among connected minima for $N=17$;

5. for each pair of directly connected minima we determine the energy barriers $\Delta \Phi_{b a r}$. from the value of the distance $d_{a b}: \Delta \Phi_{b a r}=A d^{\alpha}\left(A \simeq 10^{5}\right.$ and $\alpha \simeq 3.7$, as determined for $N=17$ system, Fig. 5 );

6 . we assign a curvature value $c_{\text {sad. }}=\left|\operatorname{det} \Phi_{\text {sad. }}^{\prime \prime}\right|$ and a down "frequency" $\tilde{\omega}_{\text {sad }}$. to each saddle point, from bivariate distribution.

We obtain in this way a set of parameters that describe the model. In order to have a good statistic description we considered different extractions of the parameters and the measured quantities were obtained by averaging over the extractions.

\section{A. Test}

Before studying the dynamical properties of the model, we concentrate on the static behavior obtained as asymptotic solution of the master equation. In this static regime we can determine, in a statistical mechanical approach, the configurational partition function

$$
\mathcal{Z}(\beta)=\int d^{3 N} r \exp \left[-\beta \Phi\left(\vec{r}_{1}, \ldots, \vec{r}_{N}\right)\right],
$$

By using the approximation based on the hypothesis of short time local harmonic vibrations around a minimum, and long time collective jumps among different minima, we obtain

$$
\mathcal{Z}(\beta) \sim \sum_{a} \mathcal{Z}_{a}^{(\text {harm. })}(\beta) \exp \left[-\beta \Phi_{a}\right],
$$

where $a$ labels the minima and $\mathcal{Z}_{a}^{(\text {harm. })}$ is the contribution of harmonic vibrations around minimum $a$. This form of the configurational partition function emerges in the model as the exact infinite-time limit. The harmonic term is easy to calculate, being a $3 N$-dimensional Gaussian integral:

$$
\begin{aligned}
\mathcal{Z}_{a}^{(\text {harm. })}(\beta) & =\int d \underline{r} \exp \left[-\frac{\beta}{2} \underline{r} \Phi_{a}^{\prime \prime} \underline{r}\right] \\
& =(2 \pi)^{3 N / 2} \beta^{-3 N / 2}\left(\operatorname{det} \Phi_{a}^{\prime \prime}\right)^{-1 / 2}
\end{aligned}
$$

where $\underline{r}=\left(r_{1}, \ldots, r_{3 N}\right)$, and $\underline{r} \Phi_{a}^{\prime \prime} \underline{r}=\sum_{l, m} r_{l}\left(\Phi_{a}^{\prime \prime}\right)_{l m} r_{m}$. We then obtain the approximated partition function as:

$$
\mathcal{Z}(\beta) \sim c \beta^{-3 N / 2} \sum_{a}\left(\operatorname{det} \Phi_{a}^{\prime \prime}\right)^{-1 / 2} \exp \left(-\beta \Phi_{a}\right)
$$

from which the thermodynamical quantities can be derived, for example for the energy: $E(\beta)=-\partial_{\beta} \ln \mathcal{Z}$. To check the reliability of the model we compare the quantities calculated from (27) with those obtained through MD computation. In Fig. 6 we show the potential energy as obtained from the model (lines) and from MD (circles). The MD data are obtained in the following way. Starting from high temperature we quench rapidly the system to low temperature, entering in a glassy state; we then increase the temperature up to liquid phase (open circles). The system is subsequently slowly cooled, entering in the supercooled regime $(100-70 \mathrm{~K})$ and obtaining at the end the crystal through a first order transition (full circles). The lines represent the energies determined from the model by taking into account all the minima (dotted line) and only the glassy ones (full line). A good quantitative agreement is obtained between MD and the model as far as the temperature is lower than about $150 \mathrm{~K}$, a temperature in the liquid phase well above the melting point $\left(T_{m} \sim 80 \mathrm{~K}\right)$. This result supports the correctness of the approximation of local-vibration / collective-jumps in the description of a glass-former at not-too-high temperature. This static test is a good starting point to extend the analysis to the dynamical regime.

\section{B. Equilibrium properties}

We now determine the dynamical equilibrium properties of the model. We denote with $\mathcal{O}(\underline{r}(t), \underline{r}(0))$ a generic observable which depends on collective coordinates $\underline{r}$ at time $t$ and at initial time $t=0$. We define the statistical average value of $\mathcal{O}$ in the model as

$$
<\mathcal{O}(t)>=\sum_{b} p_{b}^{0} \sum_{a} \mathcal{O}_{a b} p_{a}(t ; b, 0),
$$

where $\mathcal{O}_{a b}$ is the value of $\mathcal{O}$ evaluated at the minimum configurations $a$ and $b: \mathcal{O}_{a b}=\mathcal{O}\left(\underline{r}_{a}, \underline{r}_{b}\right)$. In terms of the eigenvalues and eigenvectors of the transition matrix $W$ we have: 


$$
<\mathcal{O}(t)>=\sum_{n} \exp \left(\lambda_{n} t\right) \sum_{a, b} \mathcal{O}_{a b} \alpha_{a}^{(n)} \alpha_{b}^{(n)},
$$

or in terms of the eigenvectors of the symmetric matrix $w$ :

$$
<\mathcal{O}(t)>=\sum_{n} \exp \left(\lambda_{n} t\right) \sum_{a, b} \mathcal{O}_{a b}\left(p_{a}^{0} p_{b}^{0}\right)^{1 / 2} e_{a}^{(n)} e_{b}^{(n)}
$$

In the following we report a detailed analysis of the equilibrium dynamics for a network of 400 minima, averaging over 50 different extractions of the parameters that define the model. We measure the time autocorrelation function of the stress tensor, the shear viscosity, the structural relaxation times and the mass diffusion coefficient.

We first determine the time autocorrelation functions of a structural quantity which is well defined in all minimum configurations, i.e. the off-diagonal microscopic stress tensor (7). The correlation function is

$$
C(t)=\frac{1}{3}\left[<\sigma^{z x}(t) \sigma^{z x}(0)>+(x y)+(y z)\right] .
$$

The quantity $\mathcal{O}_{a b}$ in Eq. (22) is in this case

$$
\mathcal{O}_{a b}=\frac{1}{3}\left[<\sigma_{a}^{z x} \sigma_{b}^{z x}>+(x y)+(y z)\right] .
$$

We have measured the correlation functions for different temperatures, from $T=150 \mathrm{~K}$ to $T=20 \mathrm{~K}$. In Fig. 7 we report the normalized correlation functions $C(t) / C(0)$ at different temperatures (open symbols) together with the best stretched exponential fit (lines):

$$
C(t)=C(0) \exp \left[-(t / \tau)^{\beta_{k}}\right] .
$$

Contrary to the MD computations, which result in a twostep behavior for the relaxation processes (one associated to fast local dynamics and the other to structural slow dynamics, the so called $\alpha$ structural processes), the model gives only one relaxation step, associated to the structural processes, because the model can only describes the long time behavior. The results we obtain with the present model are consistent with those of MD in the allowed region ( i.e. above $T \sim 90 \mathrm{~K}$, in order to avoid crystallization in the MD computation). In inset $(a)$ we show the temperature dependence of the stretching parameter $\beta_{k}$. It emerges that: the structural relaxation dynamics is well represented by a stretched exponential decay, and that the stretching parameter $\beta_{k}$ is strongly temperature dependent.

Both results are well supported by experimental [9] and numerical [10] observations. In our case the stretching parameters $\beta_{k}$ decreases from a value of $\sim 1$ at high temperature to $\beta_{k} \sim 0.35$ at low temperature, in agreement with experimental findings [9].

From the behavior of the correlation function $C(t)$ we can obtain information about structural relaxation time $\tau$. The values of $\tau$ (inset $(b)$ ) are determined from the stretched exponential fits. We obtain an increase of many orders of magnitude in a small temperature range, as usually found in many glass-forming liquids. However, we do not find the dramatic increase of the Vogel-TammannFulcher type expected for fragile glass-former [11]. It is possible that the observed Arrhenius behavior emerges as a peculiar property of the model, which would mean that the model is unable to capture the phenomenology of "fragility". It is, however, possible that the Arrhenius law is a genuine property of glass-forming liquids with Lennard-Jones interaction, as supported by a comparison with a MD computation in the allowed temperature range. It would be very interesting to compare the behavior obtained from the model to the "true" (in the sense of MD computation) behavior in the full temperature range (this is possible only for those system that avoid crystallization, like suitable binary mixtures).

From the time autocorrelation functions of the offdiagonal microscopic stress tensor, we can determine the shear viscosity as [7]

$$
\eta=\frac{1}{K_{B} T V} \int_{0}^{\infty} d t C(t) .
$$

Also in this case, like for the relaxation times, we find a strong increase in a small temperature range, from $\eta \sim$ $10^{-2} \mathrm{P}$ at $T=150 \mathrm{~K}$, to $\eta \sim 10^{11} \mathrm{P}$ at $T=20 \mathrm{~K}$. Also in this case we found a good agreement between the model and $\mathrm{MD}$ for $T>90 \mathrm{~K}$, giving further support to the model.

The last quantity we measured in the model was the mass diffusion coefficient. In order to find it we determine the mean square displacement

$$
\mathcal{O}(t)=\frac{1}{N}|\underline{r}(t)-\underline{r}(0)|^{2}=\frac{1}{N} \sum_{i=1}^{N}\left|\vec{r}_{i}(t)-\vec{r}_{i}(0)\right|^{2},
$$

from which we obtain the diffusion coefficient:

$$
D=\lim _{t \rightarrow \infty} \frac{\mathcal{O}(t)}{6 t} .
$$

To evaluate $D$ we use the quantity $\mathcal{O}_{a b}=d_{a b}^{2} / N$, where $d_{a b}$ is defined in (10). We observe again a strong increase with an interesting behavior not simply linear in double logarithmic scale [2].

We conclude this section by analyzing the validity of the Stokes-Einstein relation in the model. The StokesEinstein relation describes in a rigorous way the diffusive motion of a macroscopic objects in a fluids and predicts the following relation:

$$
D \propto \frac{T}{\eta} .
$$

The Stokes-Einstein relation also describes fairly well the diffusion at atomic scale in liquids at high temperatures. 
By lowering temperature, as observed in many experiments 12], one usually finds a breakdown of the Eq. (37). We found [2] that at high $T$ the model satisfies asymptotically the Stokes-Einstein relation, but upon decreasing $T$ we observe a breakdown of the relation and a fit over the lowest temperature data of the type

$$
D^{-1} \propto\left(\frac{\eta}{T}\right)^{\xi}
$$

gives the value

$$
\xi \simeq 0.28
$$

This value is in fairly good agreement with experimental results found in fragile glass-formers, like o-terphenyl [13.

\section{Off-equilibrium properties}

Although introduced to analyze the long time dynamics, the model allows an easy computation of the short time, off-equilibrium dynamics. One of the main properties of glass-formers is the very strong increase of characteristic relaxation times when temperature is lowered. If these times become comparable to observational times, the system is no more able to explore the full accessible phase space and then to reach the thermal equilibrium. The observed quantities are characterized by off-equilibrium processes. In this regime the one-time quantities, such as energy or time correlation functions with fixed initial time, can no longer describe the physics of the system. The usual translational time invariance, valid in the equilibrium regime, is no more satisfied. One of the most interesting consequences of that is the fact that the fluctuation-dissipation relation does not longer hold [14]. We concentrate here on this property.

Let be $H$ the Hamiltonian of the system and $\mathcal{O}$ a generic observable dependent on microscopic variables. We define the two time autocorrelation function

$$
C\left(t, t_{w}\right)=<\mathcal{O}(t) \mathcal{O}\left(t_{w}\right)>
$$

where we suppose $t>t_{w}$ and $<\ldots>$ now means a dynamical average over initial conditions. We also introduce the response function to a perturbation $\epsilon$, which is coupled to the observable $\mathcal{O}$ and gives rise to a pertutbed Hamiltonian:

$$
H^{\prime}=H+\epsilon(t) \mathcal{O}
$$

The response is defined as

$$
R\left(t, t_{w}\right)=\left.\frac{\delta<\mathcal{O}(t)>}{\delta \epsilon\left(t_{w}\right)}\right|_{\epsilon=0},
$$

where again $t>t_{w}$. In the equilibrium regime the time translational invariance implies the validity of fluctuation dissipation relation 15]

$$
R_{e q}(\tau)=\beta \frac{\partial C_{e q}(\tau)}{\partial \tau},
$$

where $\tau=t-t_{w}$. Introducing the integrate response function $\chi$

$$
\chi\left(t, t_{w}\right)=\int_{t_{w}}^{t} d t^{\prime} R\left(t, t^{\prime}\right),
$$

Eq. (43) takes the form

$$
\frac{d \chi(C)}{d C}=\beta
$$

In the off-equilibrium regime the fluctuation-dissipation ratio (45) is no more valid. It is possible however to generalize the ratio introducing a violation factor $X\left(t, t_{w}\right)$. The analytical study of some generalized mean field spin glass models [16] shows that the function $X\left(t, t_{w}\right)$ depends on time only through the correlation function $C$ : $X\left(t, t_{w}\right)=X\left[C\left(t, t_{w}\right)\right]$. Using this property we can write a generalized fluctuation-dissipation ratio in the off-equilibrium regime

$$
\frac{d \chi(C)}{d C}=\beta X(C) .
$$

For short times $\tau \ll t_{w}$ we have $X(C)=1$ and the system satisfies an equilibrium-like relation, even if it is confined in a small phase space region. For times $\tau \sim t_{w}$ the exploration of the phase space is an offequilibrium process and this implies the violation of the equilibrium fluctuation-dissipation ratio. In this case we have $X(C)<1$. The very interesting relationship between off-equilibrium and equilibrium properties of some generalized spin glass model, suggests that in the case of one step replica symmetry breaking the $X(C)$ function depends only on temperature

$$
X(C)=m(T)
$$

and $m(T)$ is linear in $T$ at low temperature. It has been recently suggested that structural glasses present a striking similarity with the generalized spin glass model with one step replica symmetry breaking [17] (for a recent interesting review see Coluzzi [19]). We then expect that also for structural glasses the violation parameter would show a linear temperature dependence in the violation region $X<1$. Evidence of this behavior was found in recent numerical study of binary mixtures [18]; we analyze it in our model.

Let be $\mathcal{O}(\underline{r}(t))$ a generic observable that depends on collective coordinates at time $t$. The average value of $\mathcal{O}$ in the off-equilibrium regime in the model is 


$$
<\mathcal{O}(t)>=\frac{1}{M^{\prime}} \sum_{a, b} \mathcal{O}_{a} p_{a}(t ; b, 0)
$$

where $a$ and $b$ label the minima and the sum over $b$ is now limited to a certain subset of minima. We chose the $M^{\prime}$ highest energy states. Expression (48) differs from the equilibrium one, as the initial states are weighted with a constant term (corresponding to an infinite temperature) rather than with the Gibbs-Boltzmann equilibrium weight. In this way we describe an instantaneous quench at time $t=0$ from $T=\infty$ to a finite temperature $T$ (the $T$ dependence is as usual in the probability $p_{a}$ ). The sum restricted to the $M^{\prime}$ initial states with highest energies ( $M^{\prime}<M$ where $M$ is the total number of minima; in our case $M=400$ and $M^{\prime}=20$ ) allows a better description of the off-equilibrium regime. We calculate the time correlation functions in the model as

$$
<\mathcal{O}(t) \mathcal{O}\left(t_{w}\right)>=\frac{1}{M^{\prime}} \sum_{a, b, c} \mathcal{O}_{a} \mathcal{O}_{b} p_{b}\left(t_{w} ; c, 0\right) p_{a}\left(t ; b, t_{w}\right)
$$

where the sum over $b$ is still made over the $M^{\prime}$ highest minima. The quantity we determine is the time autocorrelation function of the off-diagonal microscopic stress tensor $\sigma^{z x}$ :

$C\left(t, t_{w}\right)=<\sigma^{z x}(t) \sigma^{z x}\left(t_{w}\right)>-<\sigma^{z x}(t)><\sigma^{z x}\left(t_{w}\right)>$.

The response function is determined by the perturbed Hamiltonian

$$
H^{\prime}=H+\epsilon(t) \sigma^{z x},
$$

where the external field $\epsilon$ is

$$
\epsilon(t)= \begin{cases}0 & \text { for } t<t_{w} \\ \epsilon & \text { for } t \geq t_{w} .\end{cases}
$$

The perturbation induces a change in the energies of the minima:

$$
\Phi_{a}^{\prime}=\Phi_{a}+\epsilon(t) \sigma_{a}^{z x}
$$

The response function is

$$
R\left(t, t_{w}\right)=\left.\frac{\delta<\sigma^{z x}(t)>_{\epsilon}}{\delta \epsilon\left(t_{w}\right)}\right|_{\epsilon=0} .
$$

The $\langle\ldots\rangle_{\epsilon}$ is evaluated in the presence of the perturbation $\epsilon$ :

$$
<\sigma^{z x}(t)>_{\epsilon}=\frac{1}{M^{\prime}} \sum_{a, b, c} \sigma_{a}^{z x} p_{b}\left(t_{w} ; c, 0\right) p_{a}^{\epsilon}\left(t ; b, t_{w}\right),
$$

where $p^{\epsilon}$ is the solution of the master equation with the perturbing term. For small perturbation we obtain:

$$
\chi\left(t, t_{w}\right)=\frac{\left.\left.<\sigma^{z x}(t)\right)>_{\epsilon}-<\sigma^{z x}(t)\right)>_{\epsilon=0}}{\epsilon} .
$$

We have determined the correlation functions $C\left(t, t_{w}\right)$ and the response $\chi\left(t, t_{w}\right)$ as functions of $t$ for different times $t_{w}$; the temperatures we analyze are in the range $T=100 \div 20 \mathrm{~K}$. In determining the response functions we have used a value of $\epsilon$ small enough $(\epsilon=0.1)$ that the regime is linear, as verified by trying different $\epsilon$ values. In Fig. 8 we report the behavior of $\chi$ versus $\beta C$ at temperatures $T=90 \mathrm{~K}$ and $T=45 \mathrm{~K}$, respectively. While at $T=90 \mathrm{~K}$ the relation between $\chi$ and $\beta C$ is to a good approximation linear with slope 1 on the whole range (full line), at lower temperature it is evident that after a first linear behavior with slope 1 (full line) an approximately linear behavior with slope $<1$ takes on at longer times (dashed line), as theoretically and numerically expected. Moreover the slope of the second region decreases by lowering temperature: in Fig. 9 we show the slope $m$ of the violation region versus $T$. At high temperature the value of $m$ is nearly 1 , while below a temperature of about $60 \div 70 \mathrm{~K} m$ decreases linearly, as we expect in the hypothesis of one step replica symmetry breaking. Fig. 9 is limited to $T>40 \mathrm{~K}$, as for lower temperatures the $m$ values saturate to a limiting value and it is no more possible to extract correct information. This effect is probably due to the finite size of the system, because the sampling of the initial off-equilibrium states is not exhaustive $\left(M^{\prime}=20\right)$. In the equilibrium analysis the finite size effects do not show up in the temperature range explored, as the sampling of the initial states is complete $\left(M^{\prime}=M\right)$.

In conclusion from the analysis of the off-equilibrium properties of the model it emerges that the deviation from the usual fluctuation-dissipation relation, valid in the equilibrium regime, is in agreement with theoretical predictions and numerical findings in simple glassformers.

\section{CONCLUSIONS}

The very rich phenomenology of the cooling process of glass-forming liquids, of the glass transition and of the glassy systems in general, has received in the last few years many important theoretical, numerical and experimental contributions. The present work is concerned with the numerical investigation of a simple model glass, a Lennard-Jones system of interacting particles. The main aim of the work was to determine the emergent properties of the system at the level of potential energy landscape. After a detailed analysis of the topological properties of the potential energy surface, we introduced a model which reproduces the long time dynamic behavior of the system. While in the usual MD investigations of relaxation the computational times are proportional 
to physical times (with computational times of the order $10^{5} \mathrm{~s}$ one obtains physical times of the order $10^{-9} \mathrm{~s}$ for system of size $N \sim 10^{3}$ ), our model allows the study at very long physical times in short computational times.

We studied both equilibrium and off-equilibrium properties. The main equilibrium results we obtained are $(i)$ the stretching of the relaxation dynamics, (ii) the temperature dependence of the stretching parameter, and (iii) the breakdown of the Stokes-Einstein relation. If they are genuine properties of the glassy system analyzed, they represent intriguing and interesting results that open fascinating questions about the glassy and supercooled liquid behavior.

Although introduced to investigate the long time dynamics, the model is also able to describe in a simple and direct way the off equilibrium dynamics. The emergent violation of the fluctuation-dissipation relation (that holds at equilibrium) is a very interesting feature and supports many conjectures about the analogy between structural glasses and some spin glass model [17]. Moreover, the appearance of a critical temperature below which the violation takes place, seems to indicate the existence of a transition; its relation with the equilibrium properties is an open and exciting question.

In conclusion, the analyzed features of the potential energy landscape and the emergent properties of the model both at and off equilibrium, seem to provide a good description of the glassy systems. The method is very powerful for the investigation of the glassy properties by avoiding some of the main problems usually encountered in numerical studies, like the very long computational times in the low temperature regime or the presence of crystal states. We hope the analysis we performed may constitute a promising route in the investigation of glassy systems.

We acknowledge B. Coluzzi, G. Monaco, F. Sciortino and P. Verrocchio for useful discussions, and D. Leporini who kept our attention on the fractional SE relation issue.

[1] M. Goldstein, J. Chem. Phys. 51, 3728 (1969); G. Adam and J.H. Gibbs, J. Chem. Phys. 57, 470 (1972); F.H. Stillinger and T.A. Weber, Phys. Rev. A 28, 2408 (1983). F.H. Stillinger, Science 267, 1935 (1995); J.P.K. Doye and D.J. Wales, J. Chem. Phys. 105, 8428 (1996); C.A. Angell, Nature 393, 521 (1998); S. Sastry, P.G. Debenedetti and F.H. Stillinger, Nature 393, 554 (1998).

[2] L. Angelani, G. Parisi, G. Ruocco, and G. Viliani, Phys. Rev. Lett. 81, 4648 (1998).

[3] M.P. Allen and D.J. Tildesley, Computer Simulation of Liquids, Oxford University Press (1987).
[4] T.B. Schroder, S. Sastry, J.C. Dyre and S.C. Glotzer, cond-mat/9901271

[5] F. Demichelis, G. Viliani and G. Ruocco, condmat/9804114.

[6] G. Daldoss, O. Pilla and G. Viliani, Philos. Mag. B 77, 693 (1998); G. Daldoss, O. Pilla, G. Viliani, G. Ruocco, and C. Brangian, cond-mat/9804113.

[7] U. Balucani and M. Zoppi, Dynamics of the Liquid State, Oxford University Press (1994).

[8] H. Risken, The Fokker-Planck Equation, Springer-Verlag, Berlin (1984); P. Hanggi, J. of Stat. Phys. 42, 105 (1985).

[9] M.T. Cicerone, F.R. Blackburn, and M.D. Ediger, J. Chem. Phys, 102, 471 (1995).

[10] S. Sastry, P.G. Debenedetti, and F.H. Stillinger, Nature 393, 554 (1998); W. Kob and H.C. Andersen, Phys. Rev. E 52, 4134 (1995).

[11] C.A. Angell, Science 267, 1924 (1995).

[12] R. Kind et al., Phys. Rev. B 45, 7694 (1992); F. Fujara et al., Z. Phys. B 88, 195 (1992); I. Change et al., J. Non-Cryst Solids, 172-174, 248 (1994); F.R. Blackburn et al., J. Non-Cryst Solids, 172-174, 256 (1994).

[13] L. Andreozzi, A. Di Schino, M. Giordano and D. Leporini, J. Phys.: Condens. Matter 8, 9605 (1996).

[14] J.P. Bouchaud, L.F. Cugliandolo, J. Kurchan and M. Mezard, Out of equilibrium dynamics in spin glasses and the other glassy systems, in Spin Glasses and Random Fields, edited by P. Young, World Scientific (1997); cond-mat/9702070.

[15] G. Parisi, Statistical Field Theory, Addison-Wesley Publishing Company (1988).

[16] L.F. Cugliandolo and J. Kurchan, Phys. Rev. Lett. 71, 173 (1993); Philos. Mag. 71, 501 (1995); J. Phys. A: Math. Gen. 27, 5749 (1994).

[17] T.R. Kirkpatrick and P.G. Wolynes, Phys. Rev. B 35, 3072 (1987); T.R. Kirkpatrick and D. Thirumalai, Phys. Rev. B 36, 5388 (1987); L. Cugliandolo and J. Kurchan, Phys. Rev. Lett. 71, 173 (1993); S. Franz and G. Parisi, Phys. Rev. Lett. 79, 2486 (1997); G. Parisi, cond-mat/9712079, cond-mat/9701034; B. Coluzzi and G. Parisi, cond-mat/9712261.

[18] G. Parisi, Phys. Rev. Lett. 79, 3660 (1997); J.L. Barrat and W. Kob, cond-mat/9806027.

[19] B. Coluzzi, Phd Thesis (Rome, 1998) 
TABLE I. Correlation coefficients $r$ for the measured quantities $x$ and $y$.

\begin{tabular}{||c|c|c|c||}
\hline \hline$N$ & $x$ & $y$ & $r$ \\
\hline \hline 29 & $\Phi_{a}$ & $c_{a}$ & 0.13 \\
\hline 29 & $\Phi_{a}$ & $\gamma_{a}$ & 0.46 \\
\hline 29 & $\left|\Phi_{a}-\Phi_{b}\right|$ & $d_{a b}$ & 0.12 \\
\hline 29 & $\ln \left|\Phi_{a}-\Phi_{b}\right|$ & $\ln d_{a b}$ & 0.17 \\
\hline 17 & $\left|\Phi_{a}-\Phi_{b}\right|$ & $d_{a b}$ & 0.18 \\
\hline 17 & $\ln \left|\Phi_{a}-\Phi_{b}\right|$ & $\ln d_{a b}$ & -0.11 \\
\hline 17 & $\Phi_{\text {sad. }}$ & $c_{\text {sad. }}$ & 0.23 \\
\hline 17 & $\Phi_{\text {sad. }}$ & $\gamma_{\text {sad. }}$ & 0.49 \\
\hline 17 & $c_{\text {sad. }}$ & $\tilde{\omega}_{\text {sad. }}$ & -0.12 \\
\hline 17 & $\gamma_{\text {sad. }}$ & $\ln \tilde{\omega}_{\text {sad. }}$ & -0.31 \\
\hline 17 & $d_{a b}$ & $\Delta \Phi_{\text {bar. }}$ & 0.47 \\
\hline 17 & $\ln d_{a b}$ & $\ln \Delta \Phi_{\text {bar. }}$ & 0.67 \\
\hline \hline
\end{tabular}

\section{FIGURE CAPTIONS}

Fig. 1 - Distribution of the potential energy (in K per particle) of the minima for the system $N=29$. In the inset the distribution of the curvature $\gamma_{\min }$.

Fig. 2 - An example of potential energy profile along the least action path $(\bullet)$ between two minima, compared with the straight path (o).

Fig. 3 - Energy distribution of the barriers along the least action paths among the minima. The inset shows the distribution of the curvature $\gamma_{\text {sad. }}$. of the saddle points.

Fig. 4 - Correlation between energies and curvatures of the minima. The highest curvature values correspond to low energy crystal minima.

Fig. 5 - Correlation between energies of the barriers and distances among minima, in a log scale. The line is the best linear fit in $\log$ scale, correspondent to a power law fit (the slop is $\alpha=3.7)$.

Fig. 6 - Potential energy versus temperature as determined from MD and from the model. The MD (o) are obtained heating the glass and $(\bullet)$ cooling the liquid. Dotted line refers to the model using all the minima, while full line using only the glassy minima.

Fig. 7 - Normalized autocorrelation functions of the off-diagonal microscopic stress tensor versus time at different temperatures, obtained from the model. In the inset $(a)$ the $T$ dependence of the stretching parameter $\beta_{K}$ and in the inset (b) the relaxation time versus $1 / T$, both obtained from the stretched exponential fit of the autocorrelation functions.

Fig. 8 - The integrate response $\chi$ versus $\beta C$ at temperatures $T=$ $90 \mathrm{~K}(\circ)$ and $T=45 \mathrm{~K}(\bullet)$. The full line is the fluctuationdissipation ratio, the dotted line is the best fit of the last points of $T=45 \mathrm{~K}$.

Fig. 9 - The slop $m$ in the region of the violation of the fluctuationdissipation ratio versus temperature. The straight line fits the data in the violation region. 


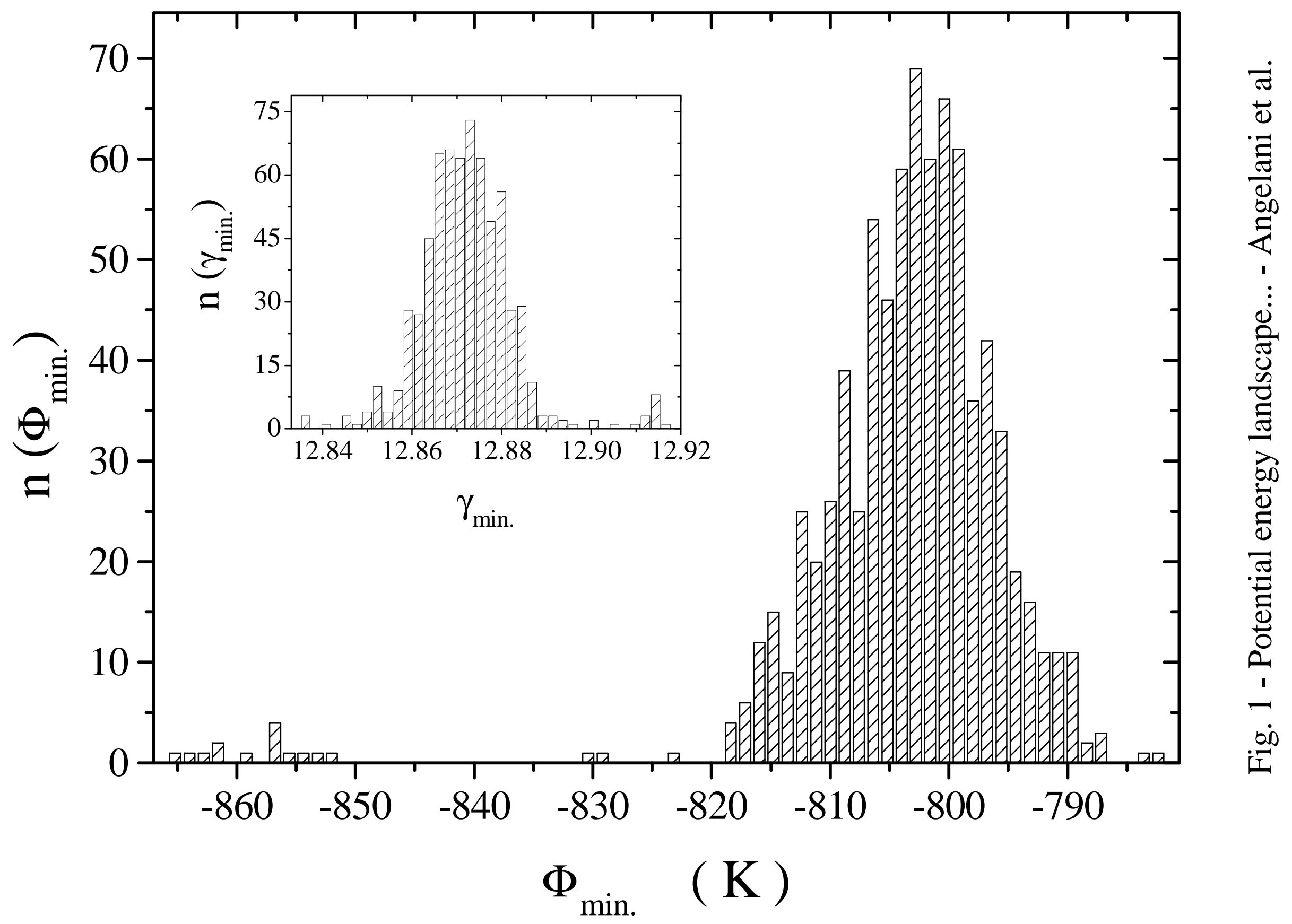


$\Phi \quad(\mathrm{K})$

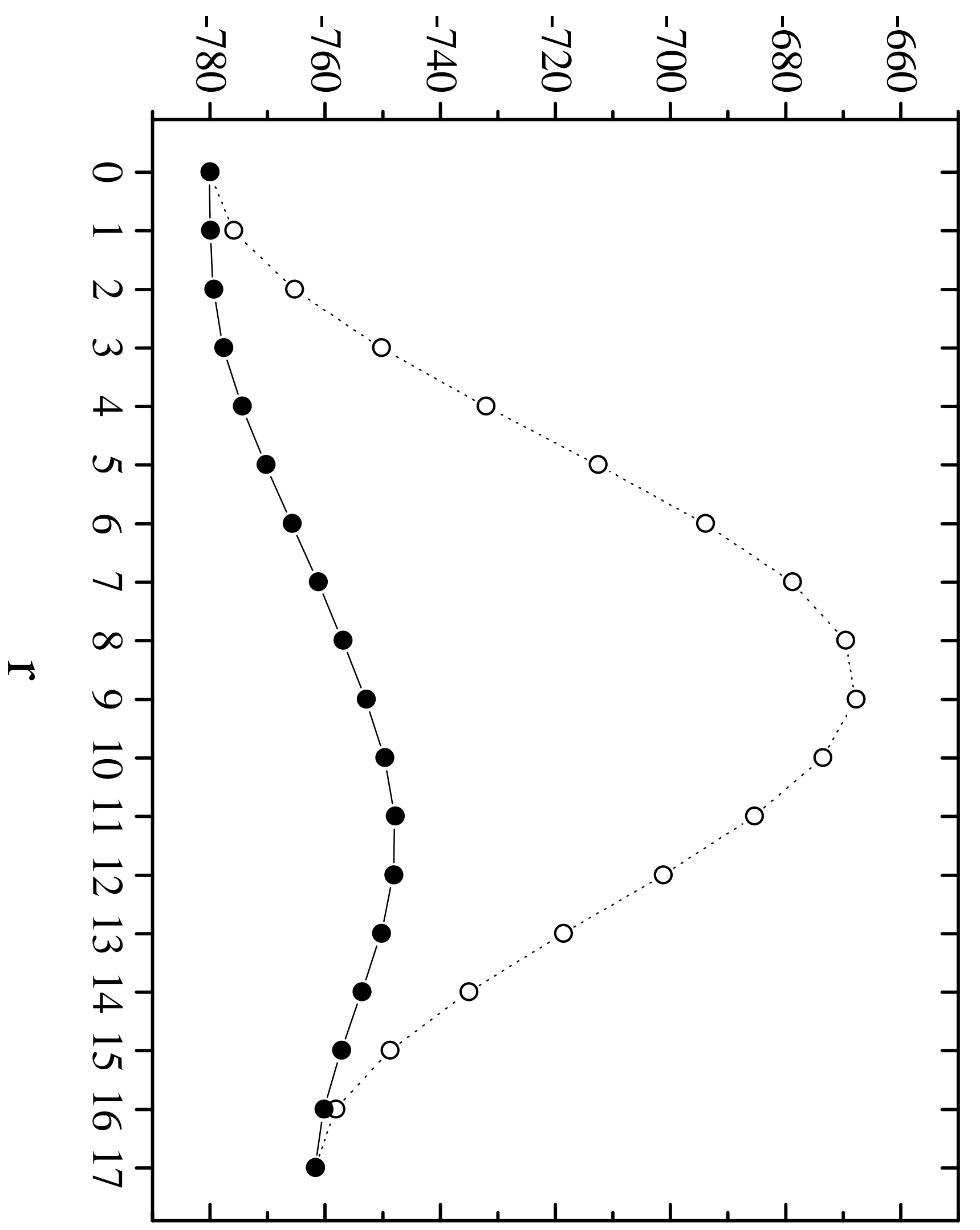

Fig. 2 - Potential energy landscape... - Angelani et al. 


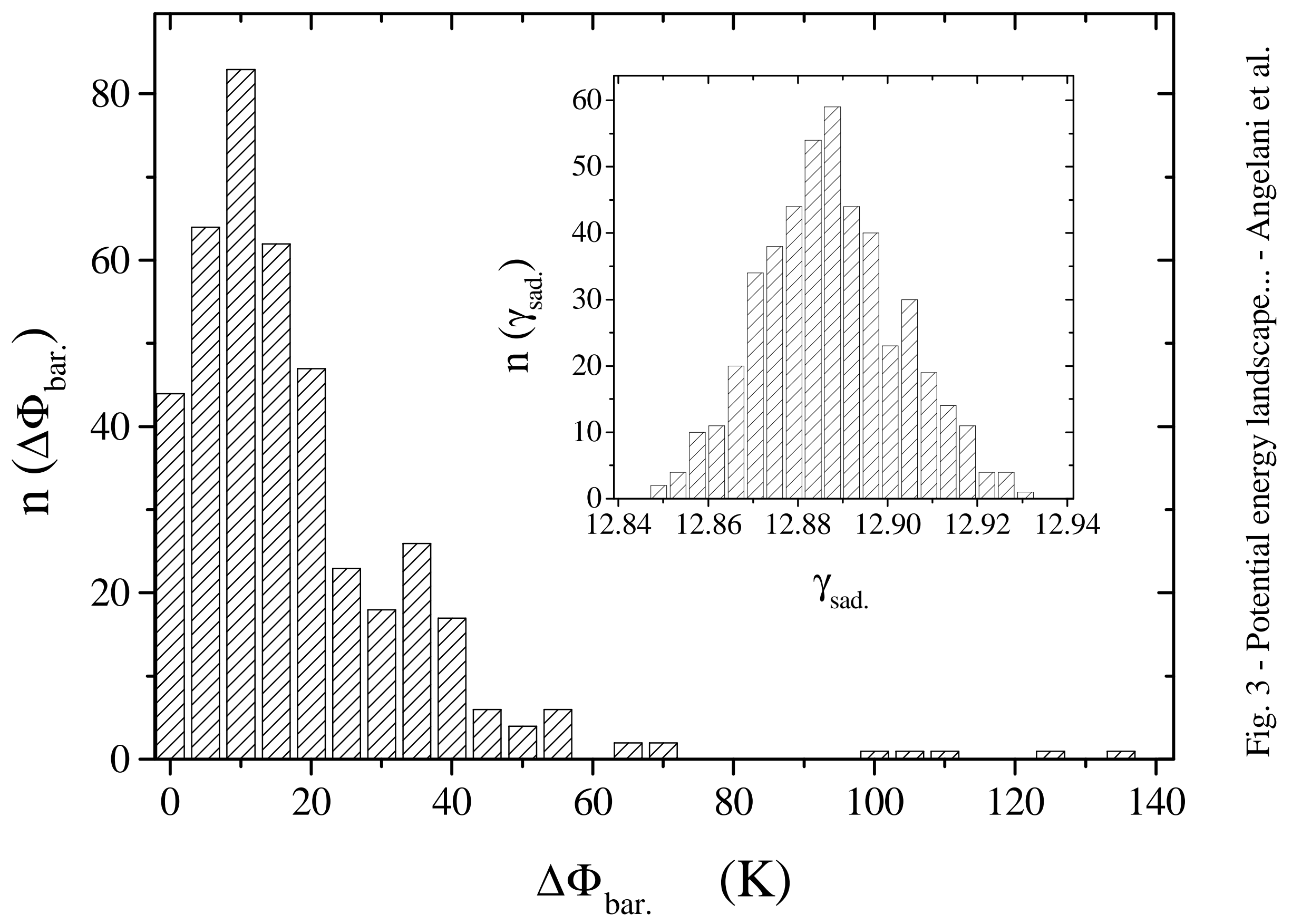




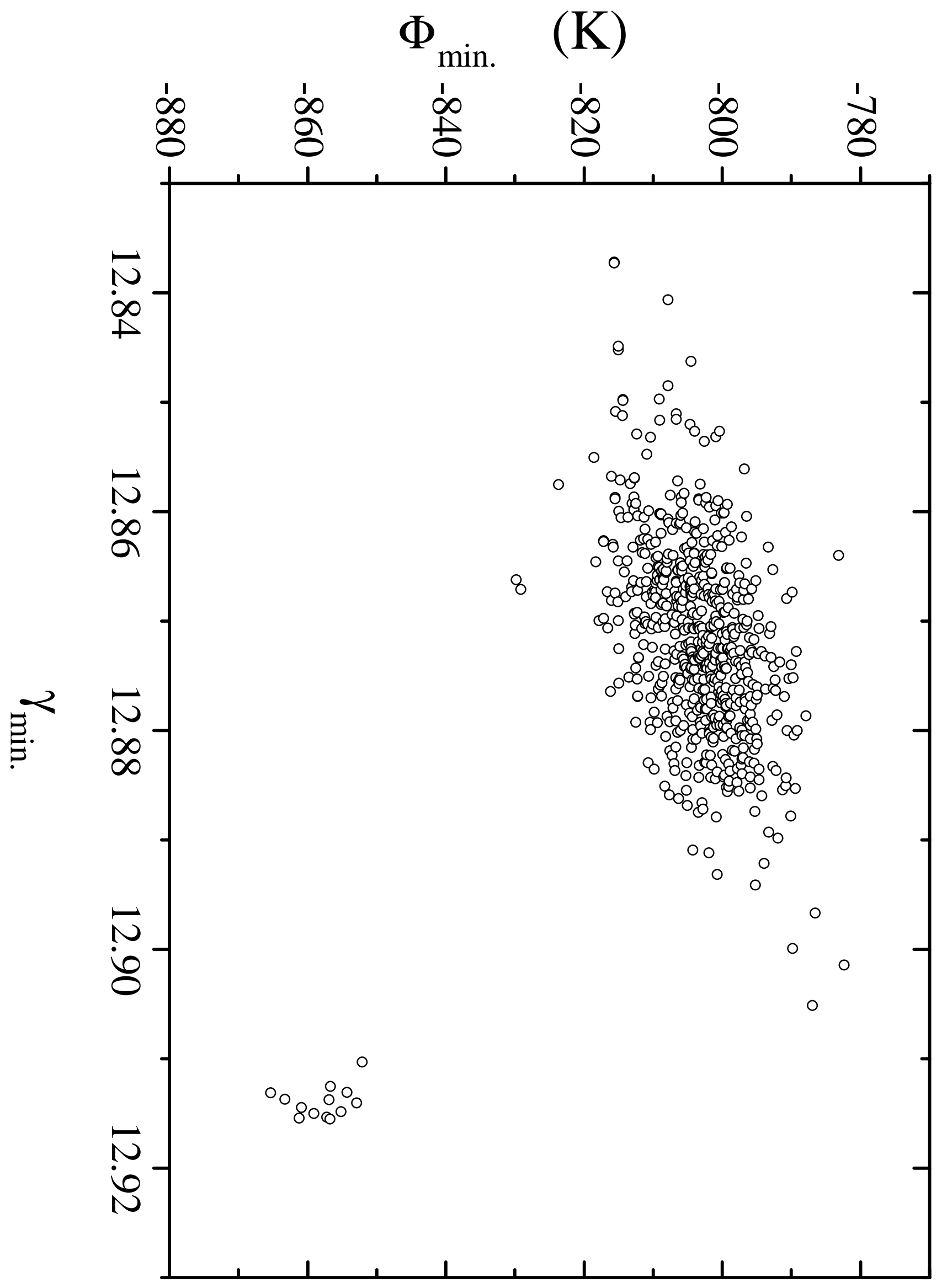

Fig. 4 - Potential energy landscape... - Angelani et al. 


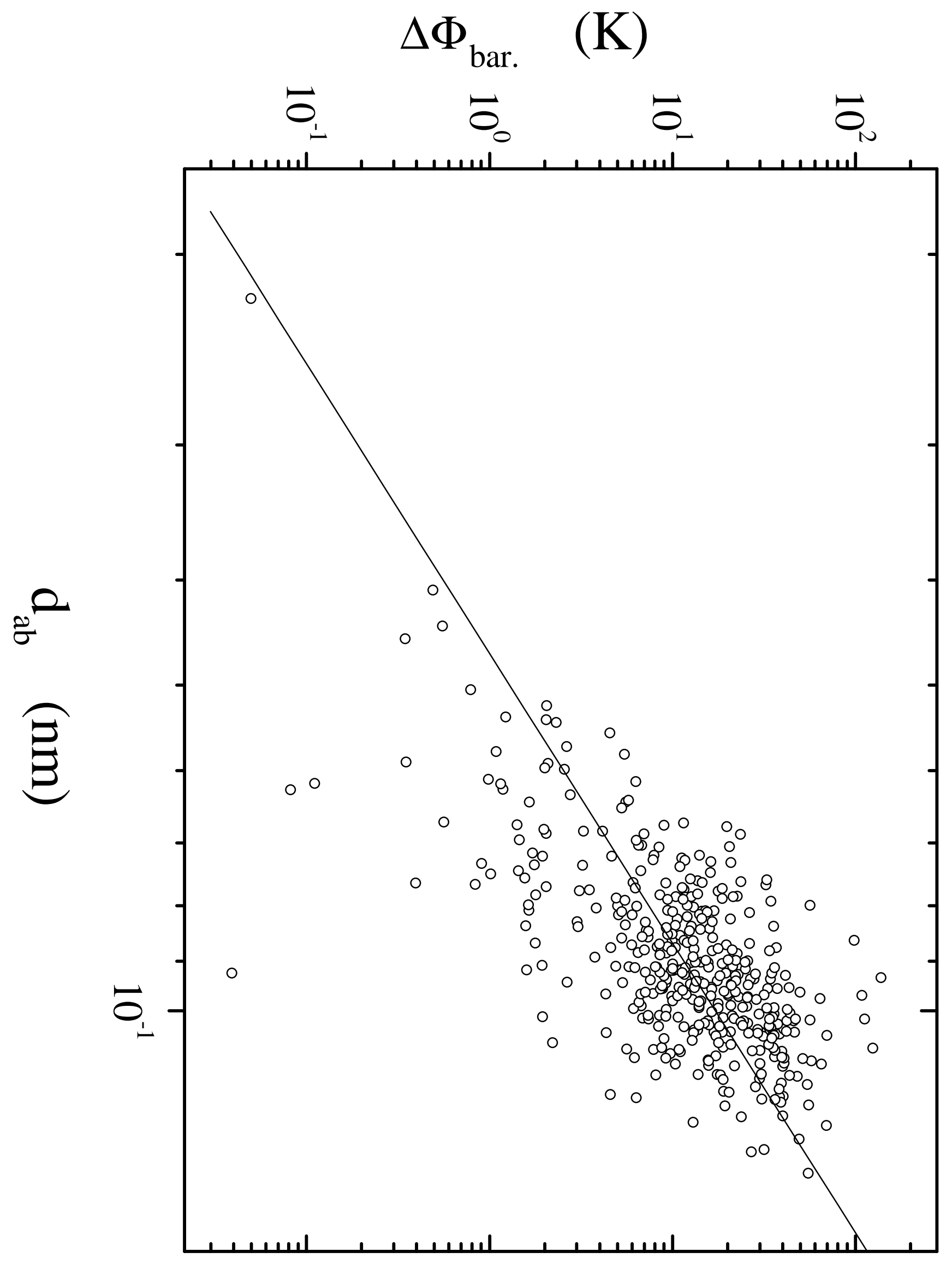

Fig. 5 - Potential energy landscape... - Angelani et al. 


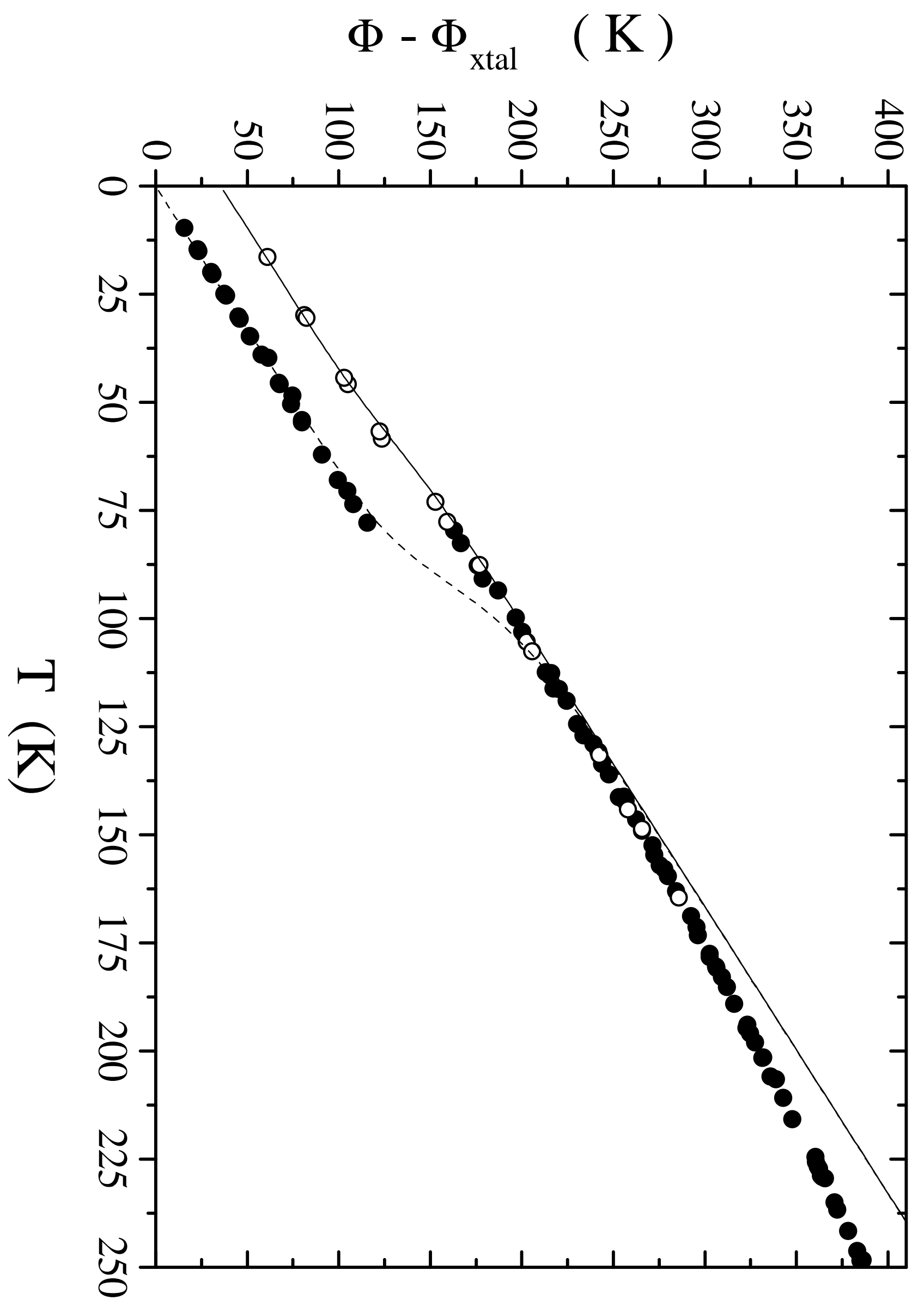

Fig. 6 - Potential energy landscape... - Angelani et al. 


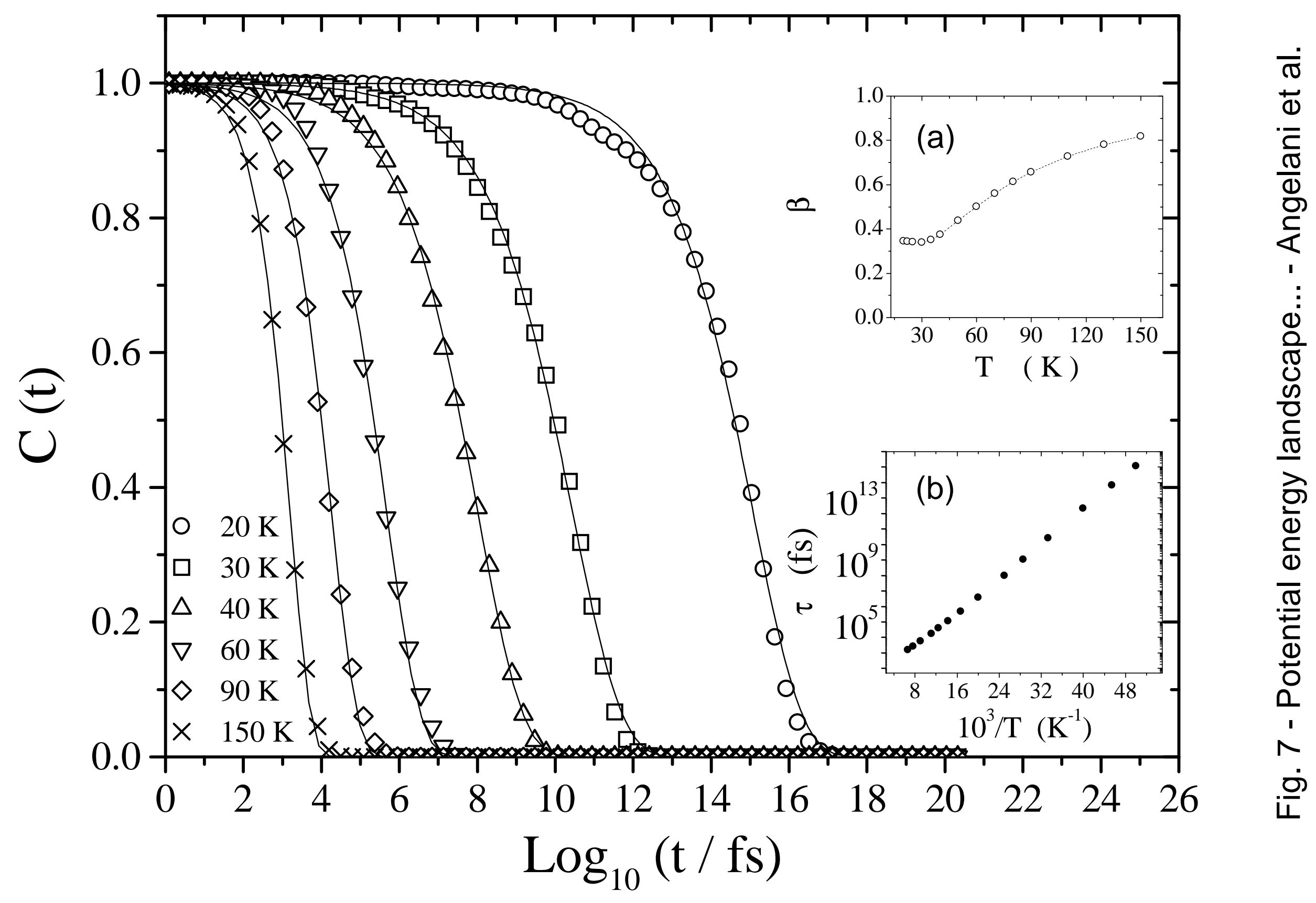




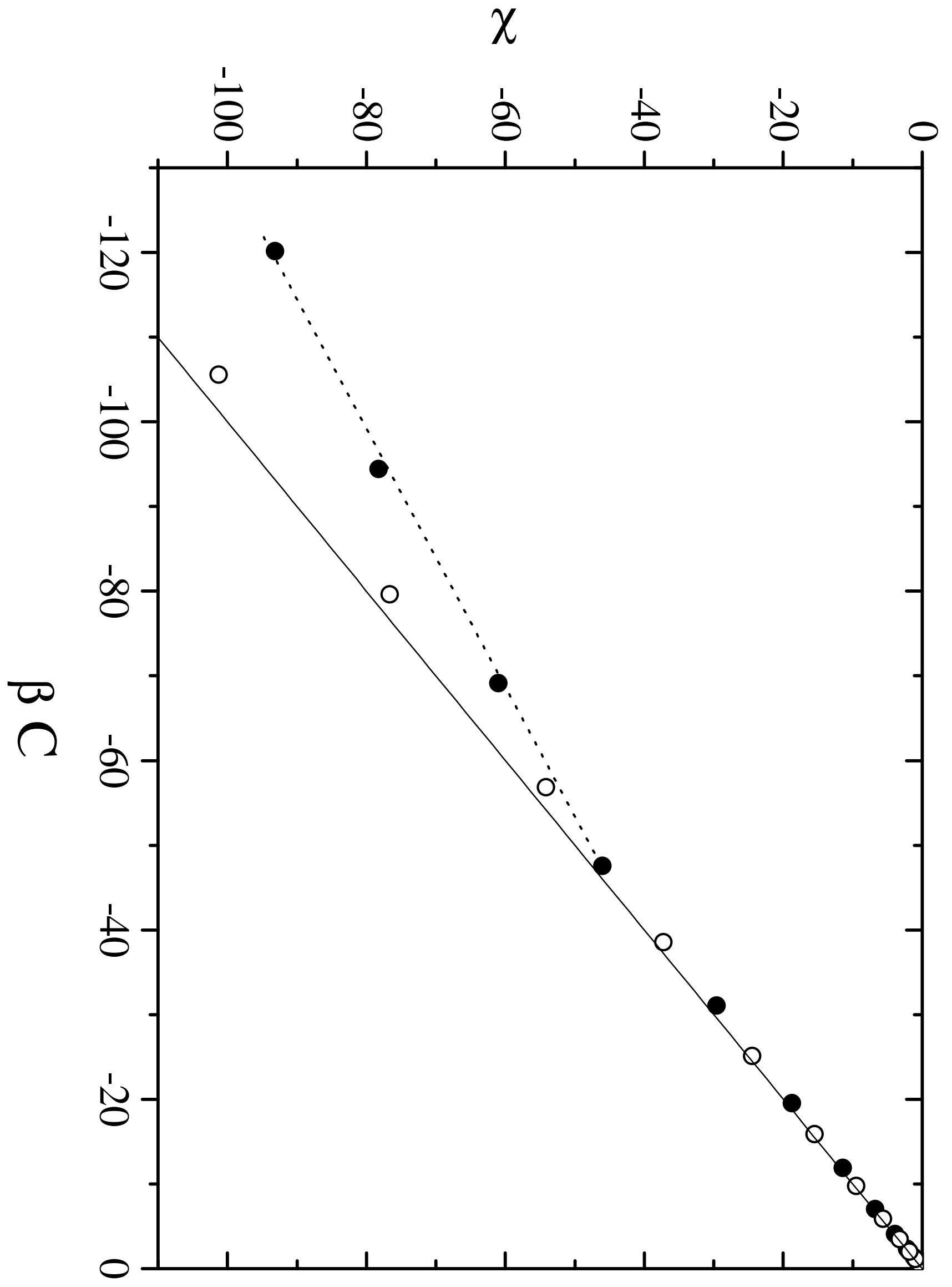

Fig. 8 - Potential energy landscape... - Angelani et al. 


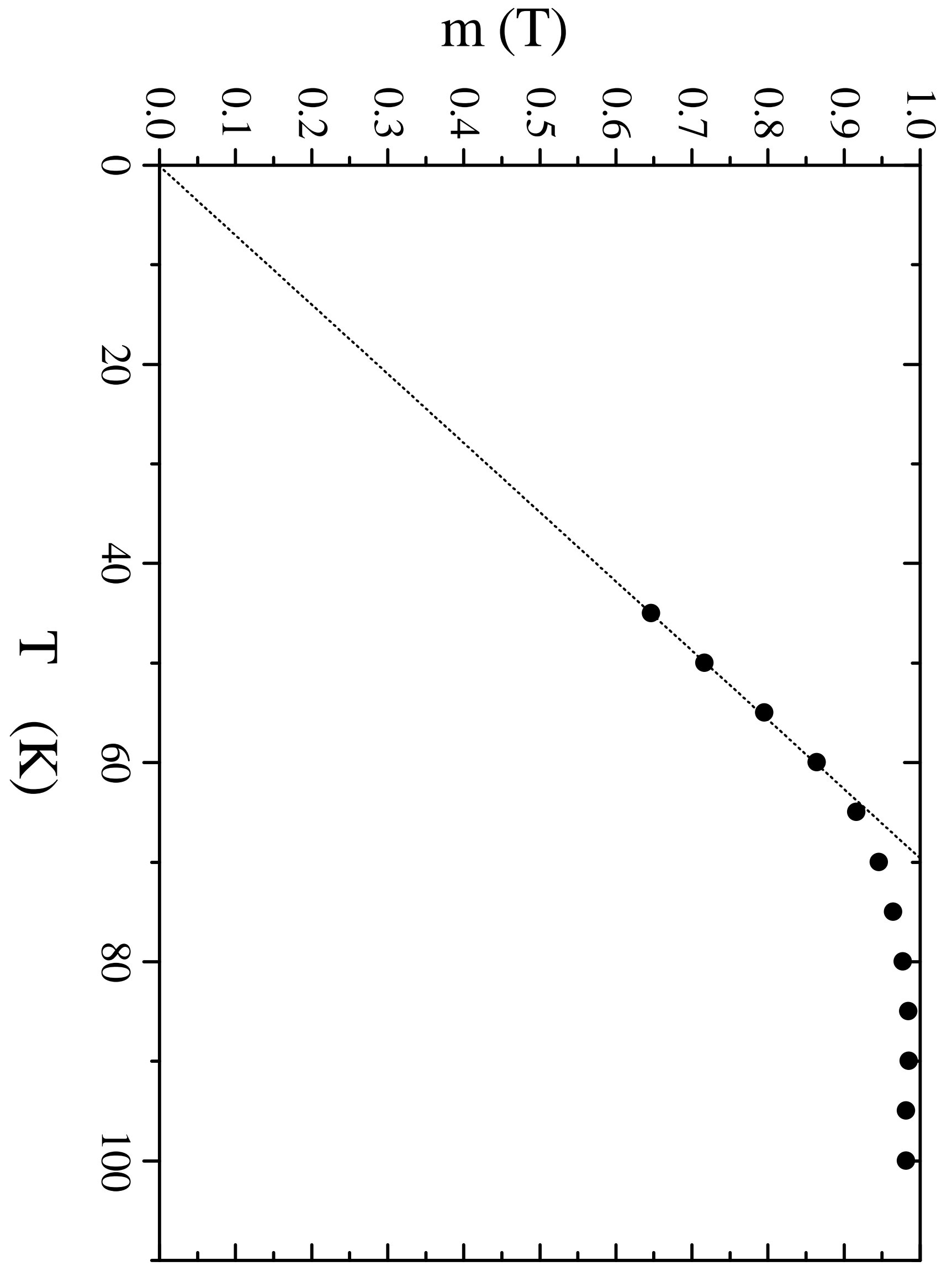

Fig. 9 - Potential energy landscape... - Angelani et al. 